\section{SEQUENCING IMMUNOTHERAPY BEFORE LYMPHATIC ABLATION UNLEASHES CDC1-DEPENDENT ANTITUMOR IMMUNITY IN HNSCC}

${ }^{1}$ Robert Saddawi-Konefka*, ${ }^{2}$ Aoife O'Farrell, ${ }^{1}$ Farhoud Faraji, ${ }^{1}$ Michael Allevato, ${ }^{1}$ Zhiyong Wang, ${ }^{1}$ Victoria Wu, ${ }^{1}$ Bryan Yung, ${ }^{1}$ Nana-Ama Anang, ${ }^{1}$ Ida Franiak-Pietryga ${ }^{3}$ Aaron Simon, ${ }^{4}$ Shawn Jensen, ${ }^{4}$ Bernard Fox, ${ }^{1}$ Andrew Sharabi, ${ }^{1}$ Ezra Cohen, 'Joseph Califano, 'J Silvio Gutkind. ' UC San Diego School of Medicine, La Jolla, CA, USA; ${ }^{2}$ University of Pennsylvania, Philidelphia, PA, USA; ${ }^{3}$ San Diego School of Medicine, San Diego, CA, USA; ${ }^{4}$ Providence Cancer Institute, Portland, OR, USA

Background Despite the proven efficacy of immune checkpoint inhibitor (ICI) therapy in the recurrent/metastatic setting for head and neck squamous cell carcinoma (HNSCC), clinical trials of ICI combined with curative-intent therapies have yielded equivocal results [1-4]. Collectively, this highlights gaps in our understanding of rational immune oncology (IO) treatment sequencing and suggests that the efficacy ICI may be disrupted by standard therapies, which necessarily compromise regional lymphatics.

Methods We employ a preclinical model of tobacco-signature HNSCC to identify sequences of therapy that maximize durable response. By mapping the cervical lymphatic basins in the mouse, we define patterns of active antitumor immunosurveillance. Additionally, we establish tumors with distinct patterns of regional lymphatic drainage and develop a murine neck dissection (ND) model.

Results We find that cervical lymphatic ablation, with ND or stereotactic body radiation therapy, in tumor bearing animals abolishes the response to ICI therapy, significantly impacting overall survival. Examination of the tumor immune microenvironment following ND reveals dramatic changes with a tenfold increase in CD45 cells and exclusion of cytotoxic and antigen-specific lymphocytes. By examining the lymphatics removed at the time of ND, we find that conventional type I dendritic cells (cDC1s) and type I interferon (IFN-I) signaling are significantly increased, suggesting that these effectors are lost after curative-intent therapy. Depleting IFN-I or cDC1s blocks the response to ICI similar to lymphatic ablation. We find that successful primary response to ICI leads to durable immunity, conferred by systemically distributed memory $\mathrm{T}$ cells, not impaired by delayed ND. Lastly, we discover a rational IO treatment sequence by delivering neoadjuvant ICI followed by ND. Neoadjuvant ICI leads to complete tumor response, accumulation of nodal $\mathrm{cDC1}$, and durable immunity. Surprisingly, the incidence of nodal metastasis at early timepoints reveals a similar burden of nodal disease between control and ICI-treated animals that decreases at late timepoints only with ICI treatment ( $44 \%$ vs $15 \%, \mathrm{n}=25, \mathrm{p}=0.033)$. This suggests that ICI also drives active immunosurveillance in regional, tumor-draining lymphatics, challenging the landmark findings from the definitive clinical trial demonstrating the benefit of elective versus therapeutic neck dissection for oral SCC patients with clinically negative necks.

Conclusions This work demonstrates the necessity of preserving tumor-draining lymphatics during the tumor response to ICI therapy in HNSCC. Overall, we define rational IO treatment sequences to achieve optimal primary tumor response, durable antitumor immunity and immunosurveillance of regional metastatic disease. These findings can inform future clinical trials investigating combination IO therapy and treatment sequencing.

\section{REFERENCES}

1. Harrington, K. J. et al. Nivolumab versus standard, single-agent therapy of investigator's choice in recurrent or metastatic squamous cell carcinoma of the head and neck (CheckMate 141): health-related quality-of-life results from a randomised, phase 3 trial. Lancet Oncology 18, 1104-1115 (2017).

2. Burtness, B. et al. Pembrolizumab alone or with chemotherapy versus cetuximab with chemotherapy for recurrent or metastatic squamous cell carcinoma of the head and neck (KEYNOTE-048): a randomised, open-label, phase 3 study. Lancet (London, England) 394, 1915-1928 (2019).

3. Lee, N. Y. et al. Avelumab plus standard-of-care chemoradiotherapy versus chemoradiotherapy alone in patients with locally advanced squamous cell carcinoma of the head and neck: a randomised, double-blind, placebo-controlled, multicentre, phase 3 trial. Lancet Oncol 22, 450-462 (2021).

4. D'Cruz, A. K. et al. Elective versus Therapeutic Neck Dissection in Node-Negative Oral Cancer. New England Journal of Medicine 373, 521-529 (2015).

http://dx.doi.org/10.1136/jitc-2021-SITC2021.601 